\title{
Kernos
}

Revue internationale et pluridisciplinaire de religion grecque antique

$11 \mid 1998$

Varia

\section{Athéna dans les cités de Macédoine}

\section{Emmanuel Voutiras}

URL : http://journals.openedition.org/kernos/1220

DOI : $10.4000 /$ kernos. 1220

ISSN : 2034-7871

Éditeur

Centre international d'étude de la religion grecque antique

Édition imprimée

Date de publication : 1 janvier 1998

ISSN : 0776-3824

\section{Référence électronique}

Emmanuel Voutiras, "Athéna dans les cités de Macédoine », Kernos [En ligne], 11 | 1998, mis en ligne le 21 avril 2011, consulté le 19 avril 2019. URL : http://journals.openedition.org/kernos/1220 ; DOI : $10.4000 /$ kernos. 1220 


\section{Athéna dans les cités de Macédoine*}

Dans l'antiquité, le terme panthéon dénote d'une façon générale l'ensemble des divinités d'un lieu ou d'une région. Cependant, dans l'étude contemporaine de la religion grecque, la notion de panthéon est souvent utilisée pour désigner un système religieux articulé, à l'intérieur duquel chaque dieu trouve sa place en fonction d'un domaine d'influence bien défini et de ses rapports avec les autres divinités ${ }^{1}$. Mais cette acception du terme, pour utile qu'elle soit comme instrument de recherche et comme principe de classification, n'est pas sans créer des difficultés ${ }^{2}$. Car les dieux grecs sont également des personnalités divines complexes se présentant sous des aspects différents, parfoịs même dans une seule région ${ }^{3}$, sans que pour autant leur identité cultuelle ou leur filiation mythologique en soient modifiées. Aux yeux des Grecs, Athéna est la déesse poliade par excellence, protectrice des acropoles, notamment de celle d'Athènes; son influence s'étend sur tout le domaine public et elle représente la sagesse et la modération dans la gestion des affaires de la cité ${ }^{4}$. Aussi l'État athénien et ses institutions sont-

* Je tiens à remercier vivement Mme Polyxeni Adam-Veleni (Musée Archéologique de Thessalonique), Mme Sophia Kremydi-Sisilianou (Athènes), Mr Ioannis Akamatis (Université de Thessalonique), M. Ioannis Touratsoglou (Musée Nu'mismatique d'Athènes) et $M$. Tzamalis (Collection Numismatique de Alpha Credit Bank, Athènes) pour m'avoir procuré des photographies et donné l'autorisation de les publier ici.

Abbréviations spéciales :

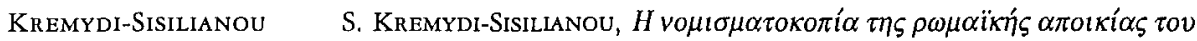

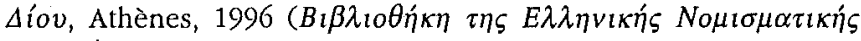

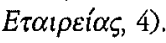

LACROIX L. LACROIX, Les reproductions de statues sur les monnaies grecques, Paris, 1949 (Bibliotbèque de la Faculté de Pbilosopbie et Lettres de l'Université de Liège, 116).

1 J.-P. Vernant, Mythe et société en Grèce ancienne, Paris, 1974, p. 104.

2 Le problème posé par cette définition est discuté par W. BurkerT, Greek Religion (trad. J. Raffan), Cambridge, Massachusetts, 1985, p. 216-21; cf. J.N. Bremmer, Götter, Mythen und Heiligtümer im antiken Griecbenland (trad. K. Brodersen), Darmstadt, 1996, p. 18-19.

3 Pour un exemple, voir M. JosT, Versions locales et versions " panbelléniques * des mythes arcadiens cbez Pausanias, in Kernos, suppl. 8 (1998), p. 227-240.

4 Sur la personnalité divine d'Athéna et la relation intime de la déesse avec Athènes, voir la belle mise au point de U. v. Wilamowitz-Monllendorff, Der Glaube der Hellenen

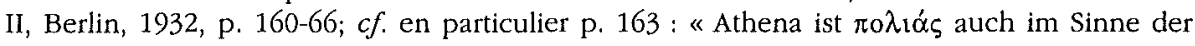
Städterin. " 
ils placés sous sa tutelle; elle est en quelque sorte la garante de la paix intérieure, de la justice et de la prospérités. Elle est aussi une redoutable guerrière qui se bat aux côtés de ses protégés pour leur assurer la victoire ${ }^{6}$. Les deux rôles, normalement complémentaires, peuvent parfois devenir contradictoires, comme on le voit par exemple dans le mythe de la guerre de Troie : pendant que la vénérable statue de la déesse, le palladion, gardée dans le temple de la citadelle d'Ilion, est censée assurer la protection de la ville, la déesse elle-même est l'alliée des Achéens et l'ennemie acharnée des Troyens. Le culte d'Athéna en Macédoine reflète ces deux aspects de la personnalité de la déesse.

Sous le règne d'Auguste, vraisemblablement entre 22 et 20 av. J.-C., la cité macédonienne de Dion en Piérie, au pied du mont Olympe, devenue colonie romaine au nom de Colonia Julia Diensis ${ }^{7}$, frappa monnaie pour la première fois dans son histoire ${ }^{8}$. Il s'agit d'un monnayage en bronze, dont la circulation était limitée presque exclusivement dans la région. La plupart de ces pièces portent au droit, comme c'est la règle dans les émissions locales sous l'empire, le portrait du princeps ${ }^{9}$. Le type du revers a un intérêt particulier, puisqu'il est en quelque sorte l'emblème de la cité : on y voit Athéna debout, vêtue du peplos attique ceint haut, sous les seins (fig. 1). Le bras gauche levé de la déesse s'appuie sur la lance tandis que la main droite tient une patère tendue en avant. La pose frontale (seule la tête est repésentée de profil, sans doute par une convention qui facilitait le travail du graveur) et la façon dont elle tient la lance et la patère font penser à une statue de culte. Ce type est d'ailleurs apparenté à certaines statues d'Athéna de la deuxième moitié du $\mathrm{VV}^{\mathrm{e}}$ siècle av. J.-C. ${ }^{10}$. L'impression qu'il s'agit d'une statue de culte est confirmée par la présence d'une base ${ }^{11}$ sur une série de monnaies de l'époque de Néron (fig. 2), où le graveur a en plus pris soin de représenter de face non seulement le corps mais aussi la tête de la déesse. Sur d'autres émissions, la base de la statue est rendue comme une simple ligne droite.

5 Cf. la fameuse élégie de Solon, fr. 4 West, avec les remarques de Wilamowitz, op. cit. (n. 2), p. 163-64.

6 Cf. HÉs., Theog., 924-26; ArchiL., fr. 94 West.

7 F. PAPAzoglou, Les Villes de Macédoine à l'époque romaine, Athènes, 1988 (BCH, Supplément XVI), p. 109; KREMYdi-Sisilianou, p. 12-14.

8 KREMYdi-Sisilianou, p. 13.

9 L'étude systématique de ces émissions n'a commencé que récemment; voir M. AMANDRY, Le monnayage des duovirs corintbiens, Athènes, 1988 (BCH, supplément XV) p. 1-2; KREMYDI-Sisilianov, p. 1-2 et 25 (avec mise au point bibliographique).

10 On peut comparer en particulier le type dit des Castra Praetoria: R. HoRN, Stebende weiblicbe Gewandstatuen frübbellenistiscber Zeit, München, 1931 (MDAIIR], Erg.-H. 2), p. 8, pl. 2,2.

11 Sur les indices qui permettent de déceler l'imitation d'une statue sur les monnaies, voir Lacroix, p. 16-22. La présence d'une base est un élément essentiel. 

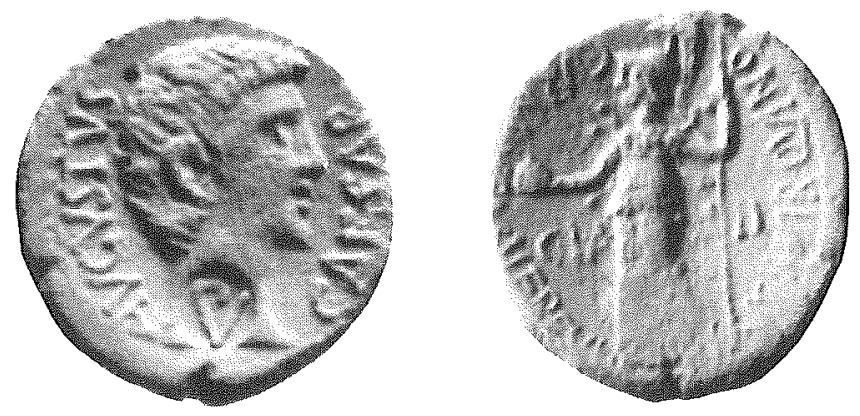

Fig. 1 : Monnaie de bronze de la colonie romaine de Dion sous Auguste.
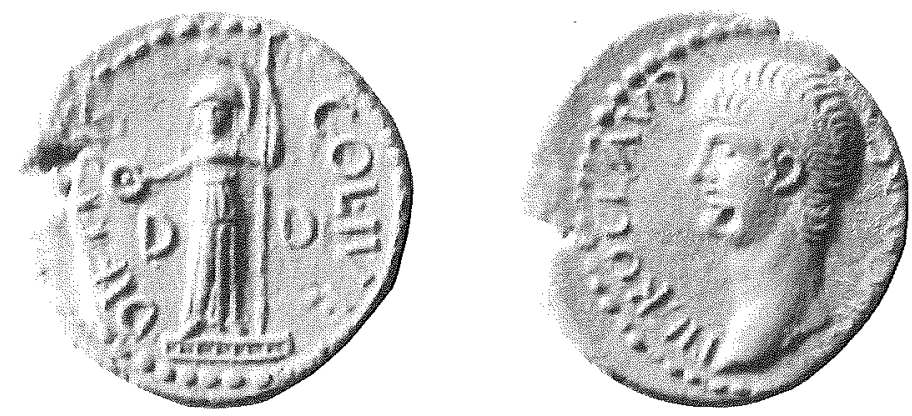

Fig. 2 : Monnaie de bronze de la colonie romaine de Dion sous Néron.

La figure d'Athéna reste de loin le type le plus répandu sur les monnaies de la colonie romaine de Dion jusqu'aux dernières émissions sous l'empereur Gallien (fig. 4). Ce choix des autorités monétaires de la colonie peut paraître, à première vue, étonnant. Il est vrai que le culte de la déesse est attesté dans la ville dès l'époque hellénistique, comme en témoigne une inscription votive, sans doute du II $^{\mathrm{e}}$ siècle av. J.-C., gravée sur un fragment d'autel ou de base circulaire provenant de la zone des sanctuaires au sud de la ville ${ }^{12}$; son importance à l'époque impériale est même mise en évidence par une inscription pour une prêtresse d'Athéna, que la ville a décidé d'honorer en érigeant sa statue $^{13}$. Cependant, le culte le plus célèbre de Dion, que l'on s'attendrait à voir commémoré sur les monnaies de la ville, était bien sûr celui de Zeus Olympios. Le sanctuaire de Zeus à Dion était en effet le plus célèbre de toute la Macédoine. Cependant, ce n'est que beaucoup plus tard, sous l'empereur

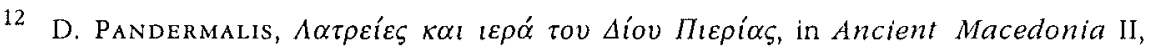
Thessalonique, 1977, p. 334; Kremydi-Sisilianou, p. 88.

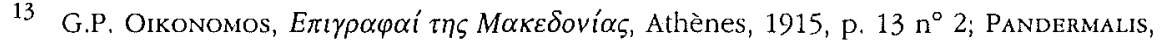
op. cit. (n. 12), p. 333-334; KREMYdi-Sisilianou, p. 88. 
Hadrien, que la figure de Zeus fait son apparition sur les monnaies de Dion ${ }^{14}$. L'introduction de ce nouveau type monétaire n'est pas difficile à expliquer : il reflète très probablement la politique d'Hadrien à l'égard de la partie hellénique de l'empire, qui fut d'emblée placée sous le signe du maître de l'Olympe ${ }^{15}$. On sait qu'Hadrien introduisit à Athènes, dans l'aire sacrée de l'Olympieion, l'ancien sanctuaire de Zeus Olympios dont il acheva le temple, le culte de Zeus Panhellénios comme centre religieux et en même temps politique ralliant toutes les cités grecques de l'empire romain ${ }^{16}$. La ville de Dion était parmi celles qui avaient dédié une statue d'Hadrien dans l'Olympieion athénien : sa base inscrite, avec dédicace en latin et le nom de la cité en grec, a été retrouvée ${ }^{17}$. Cependant, le type monétaire de Zeus n'a pas supplanté celui d'Athéna. La déesse figure même parfois à coté de son père sur les émissions de Dion à partir du règne de Marc Aurèle (fig. 3) ${ }^{18}$.
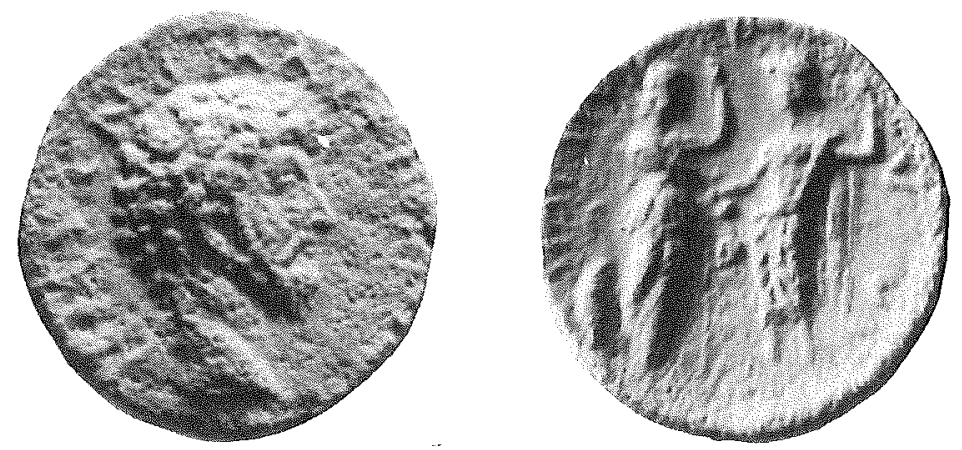

Fig. 3: Monnaie de bronze de la colonie romaine de Dion sous Marc Aurèle.

La place importante faite à Athéna dans le monnayage de Dion à l'époque impériale ne s'explique que si l'on admet qu'elle était considérée comme divinité poliade, donc protectrice de la cité ${ }^{19}$. C'est un rôle que ne pouvait assumer Zeus Olympios, le grand dieu panhellénique, qui avait été étroi-

\footnotetext{
14 Kremydi-Sisilianou, p. 89.

15 KREMYdi-Sisilianou, p. 90-91.

16 Voir P. Graindor, Atbènes sous Hadrien, Le Caire, 1934, p. 49-53. D. WiLlers, Hadrians Panbellenisches Programm, Bâle, 1990 (Antike Kunst, Beiheft 16), p. 26-67, avec la bibliographie antérieure.

17 CIL, III, $548=$ CIL, III, Suppl. 1, 7281; cf. KREMYdi-Sisilianou, p. 49 n. 47, et photographie de couverture; $I G, I^{2}$, 3289; Papazoglou, op. cit. (n. 7), p. 110 n. 39. Sur les dédicaces de statues d'Hadrien dans l'Olympieion athénien voir Graindor, op. cit. (n. 16), p. 50-51; Willers, op. cit. (n, 16), p. 48-53.
}

18 Kremydi-Sisillanou, p. 91.

19 Cf. KREMYdi-Sisilianou, p. 88. 
tement associé aux rois de Macédoine depuis l'époque classique ${ }^{20}$. On comprend dès lors qu'une présence éventuelle de la figure de Zeus sur les monnaies de la colonie romaine de Dion risquait d'évoquer un passé certes glorieux, mais auquel les nouveaux maîtres de la cité ne pouvaient se rattacher. Il n'est donc guère surprenant que les colons romains, responsables du monnayage, aient choisi comme emblème l'image d'Athéna, symbole du statut autonome de leur cité, plutôt que celle de Zeus, qui aurait évoqué l'importance de Dion comme principal centre religieux de la Macédoine et tout particulièrement de ses souverains ${ }^{21}$.
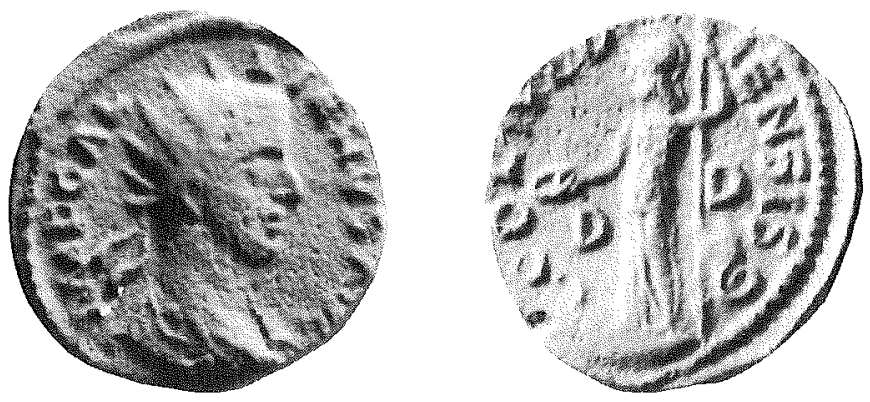

Fig. 4 : Monnaie de bronze de la colonie romaine de Dion sous Gallien.

Nous ne savons pas à quelle époque remonte le culte d'Athéna à Dion. Mais il a toute chance d'être ancien, car des sanctuaires d'Athéna sont attestés dans les cités macédoniennes dès le rv ${ }^{\mathrm{e}}$ siècle av. J.-C. Un témoignage fort intéressant semble avoir échappé aux savants qui se sont occupés des cultes de la Macédoine antique : il s'agit d'un fragment d'inscription'du $\mathbf{I V}^{\mathrm{e}}$ siècle av. J.-C. trouvé sur l'Acropole d'Athènes ${ }^{22}$. Le texte est vraisemblablement un traité entre Alexandre et la ligue hellénique renouvelant celui qui

20 On se souviendra que la tête de Zeus est très largement présente sur le monnayage de Philippe II et que la figure de Zeus assis sur son trône est de loin le type le plus commun sur les monnaies au nom d'Alexandre.

21 Cf. Kremydi-Sisilianou, p. 89-90, qui souligne justement l'importance de Zeus et en particulier de son sanctuaire à Dion pour les rois de Macédoine.

22 Publiée par A. Wilhelm, Attische Urkunden I. Teil: Urkunden des korintbischen Bundes der Hellenen, in Sitzungsbericbte Wien, 165 (6), (1911), p. 44-48, pl. IV, dont le commentaire reste fondamental; $I G, \mathrm{II}^{2}, 329$ (M.N. TOD, A Selection of Greek Historical Inscriptions, Vol. II. From 403 to 323 B.C., Oxford, 1948, ${ }^{\circ}$ 183); A.J. Heisserer, Alexander the Great and the Greeks, Norman, 1980, p. 3-26, 239, avec nouvelles restitutions et photographie (SEG, XXX [1980], 64); $c f$. K. Rosen, in Gnomon, 54 (1982), p. 354-355. Il y a lieu de noter que cette inscription fut publiée pour la première fois en 1911, l'année même de la soutenance, à Halle, de la thèse de W. BAEGE, De Macedonum sacris, qui reste, à ce jour, la seule étude d'ensemble sur le sujet. C'est probablement pour cette raison qu'elle n'est pas mentionnée dans les travaux sur les cultes de la Macédoine. 
avait été conclu par son père Philippe après la bataille de Chéronée. La dernière ligne contient une clause prévoyant l'érection d'une stèle inscrite avec ce traité dans le sanctuaire d'Athéna à Pydna, ville côtière de la Piérie et voisine de Dion, dont le port desservait la partie occidentale de la plaine

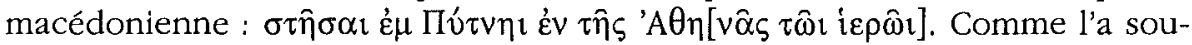
ligné à juste titre le premier éditeur, cette inscription montre que le port de Pydna jouait, à cause de sa position stratégique, un rôle important dans les relations des rois de Macédoine avec les cités grecques à l'époque classique et qu'elle était probablement le siège d'un collège chargé de surveiller le

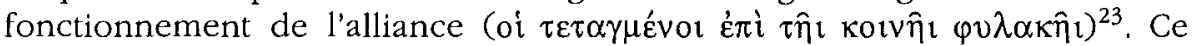
sanctuaire d'Athéna, inconnu par ailleurs, était sans doute l'un des plus importants de la ville, puisqu'on y érigeait des stèles inscrites avec des traités internationaux. Il n'est pas impossible qu'Athéna ait été la divinité principale de Pydna. Un deuxième document digne d'attention provient de Béroia. Il s'agit d'un fragment d'architrave inscrit qui nous apprend que Philippe $V$ fit construire les portiques du sanctuaire d'Athéna dans cette cité ${ }^{24}$.

Athéna était sans doute également vénérée dans d'autres cités de Macédoine, comme en témoignent des trouvailles fortuites. Ainsi, la déesse est représentée sur un relief de la basse époque hellénistique découvert en Éordée, à $2 \mathrm{~km}$ au sud d'une ville antique située sur une colline près du défilé qui mène en Lyncestide, non loin du village moderne de Pétrès, construit sur le bord méridional du lac portant son nom ${ }^{25}$. Athéna est représentée de face, debout, coiffée d'un casque, vêtue du péplos attique, la main droite levée appuyée sur la lance, la main gauche posée sur le bouclier (fig. 5). Le type iconographique, qui rappelle les monnaies de Dion, fait penser à une Athéna polias. Ce relief n'est pas une trouvaille isolée : une statue d'Artémis provenant du même champ fait supposer la présence de sanctuaires extra muros dans cette contrée ${ }^{26}$. La ville antique, que l'on ne peut pas identifier avec

23 WILHELM, op. cit. (n. 22), p. 48 : «Gerade das ist wichtig, daß zur Aufzeichnung dieser $\sigma v \vee \tau \alpha \alpha_{\xi} \varepsilon \iota \varsigma$ ein übrigens anscheinend sonst nicht bekanntes Heiligtum der Athena in Pydna gewählt ist; es liegt nahe anzunehemen, nicht nur daß der König von Makedonien die auf sein Verhältnis zu den Hellenen bezüglichen Urkunden in diesem Heiligtum der altgriechischen Hafenstadt Makedoniens aufstellen ließ, sondern auch daß Pydna, vermöge seiner Lage hierzu wie keine andere Stadt des Bundesgebiets geeignet, zugleich der

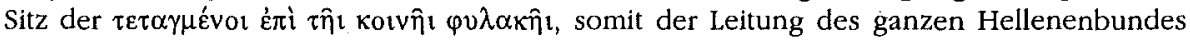
gewesen ist. " Les doutes émis récemment par A. Tronson, in AncW, 12 (1985), p. 15-19 ( $S E G, \mathrm{XXXV}$ [1985], 66), sur les conclusions de Wilhelm ne paraissent pas justifiés.

24 Ph. PETSAs, in ADelt, 20 (1965), Chronika, B3 (paru en 1968), p. 427, pl. 478b; ID., in Makedonika, 7 (1966-67), p. 321, $\mathrm{n}^{\circ}$ 165; (SEG XXIV [1969], 501; Bull. épigr. 1969, 362); M.B. Hatzopoulos, Macedonian Institutions under the Kings II. Epigraphic Appendix, Athènes, $1996(M \varepsilon \lambda \varepsilon \tau \tilde{\eta} \mu \alpha \tau \alpha, 22)$, p. 47-48, $\mathrm{n}^{\circ} 26$.

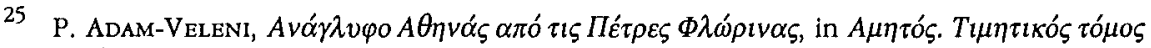

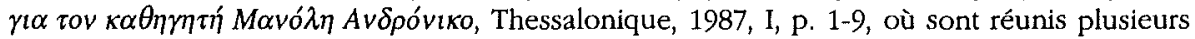
témoignages archéologiques, en partie inédits, relatifs au culte d'Athéna en Macédoine.

26

ADAM-VELENI, op, cit. (n. 25), p. 4. 


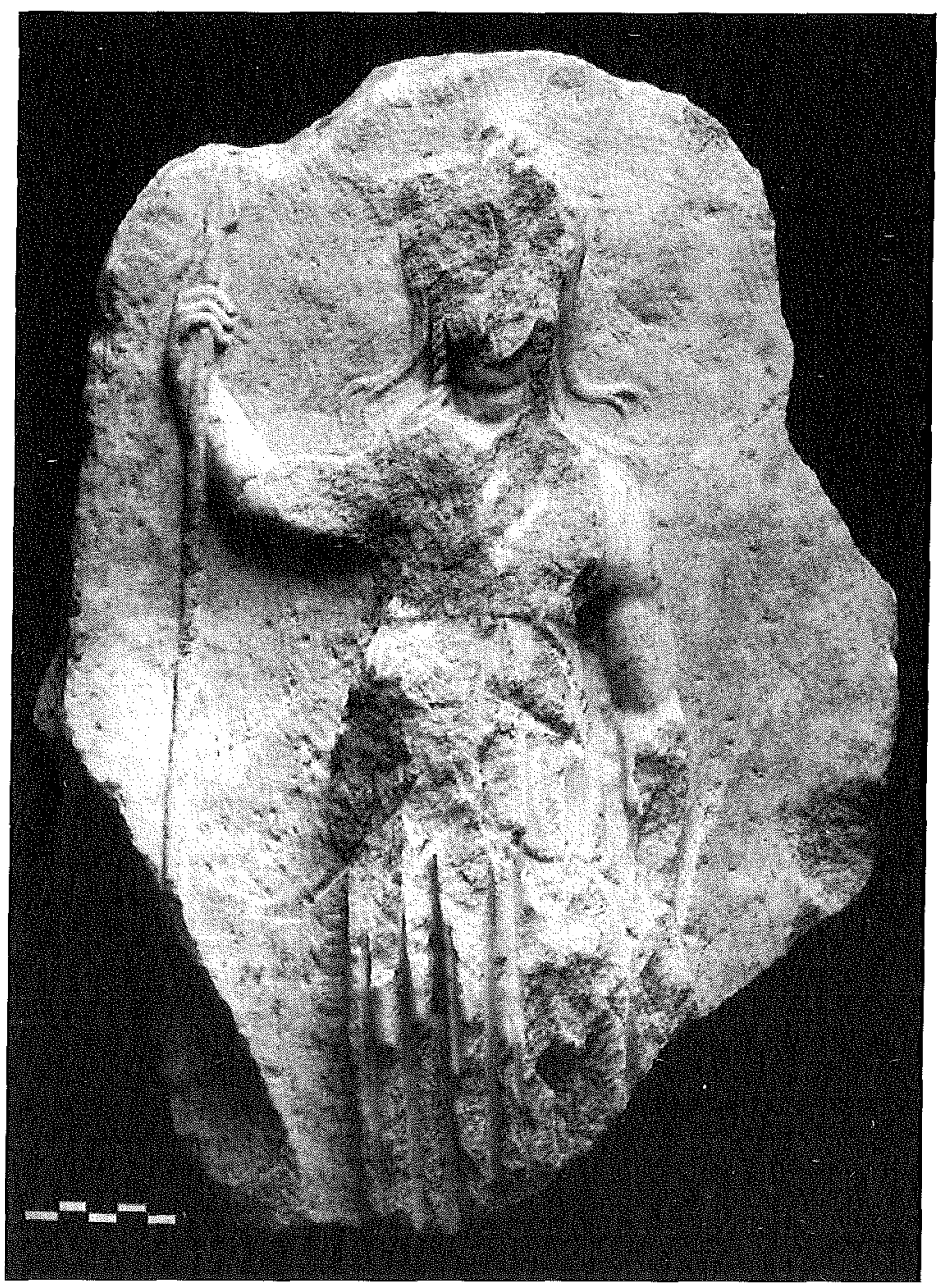

Fig. 5 : Relief votif de Pétrès (Éordée), Musée Archéologique de Florina

certitude, a connu son plus grand essor dans la seconde moitié du II $^{\mathrm{e}}$ siècle av. J.-C. Ceci s'explique sans doute par la construction de la via Egnatia, la voie militaire romaine unissant la côte de l'Adriatique à la Thrace, qui passait précisément par là ${ }^{27}$. Le relief d'Athéna appartient justement à cette période;

27 Sur tracé de la via Egnatia à travers l'Éordée et sur l'identification possible du site archéologique de Pétrès avec Arnissa voir Papazoglou, op. cit. (n. 7), p. 161-64, ainsi que 
il est donc postérieur à la conquête romaine de la Macédoine ${ }^{28}$. Son état fragmentaire ne permet pas de décider s'il était un monument votif ou s'il appartenait au décor sculpté d'un bâtiment ${ }^{29}$. Sa présence prouve néanmoins qu'Athéna faisait partie du panthéon de cette ville macédonienne et qu'elle y était probablement adorée comme divinité civique.

Mais les témoins les plus clairs de la signification politique du culte d'Athéna dans les cités sont, bien sûr, les monnaies. Le monnayage en bronze d'Amphipolis, de Thessalonique et de Pella de la période après $187 / 6$ av. J.C. ${ }^{30}$ comprend des émissions portant sur le droit la tête d'Athéna. Ce type est sans doute inspiré de celui des tétradrachmes athéniens dits de « nouveau style $»^{31}$, dont le début se place très probablement vers $170-60$ av. J.-C. ${ }^{32}$. Par conséquent, les émissions des trois cités macédoniennes à la tête d'Athéna sont à dater peu après la victoire des Romains contre Persée à Pydna en juin 168. Le choix du type monétaire devient d'autant plus significatif qu'il coïncide avec un véritable bouleversement politique auquel les cités macédoniennes se trouvèrent confrontées : la disparition du pouvoir royal. Il y a lieu de penser que le monnayage athénien a servi de modèle non seulement à cause de son prestige, mais aussi (et peut-être surtout) parce qu'il était porteur de la notion même d'autonomie civique. On observera qu'avant

les rapports sur les fouilles récentes: G. VeLENis, in AEMTh, 1 (1987), p. 9-22; G. VelENIS, P. ADAM-VELENI, AEMTh, 2 (1988), p. 5-18.

28 Voir Adam-Veleni, op. cit. (n. 25), p. 3-4.

29 ADAM-VELENI, op. cit. (n. 25), p. 8.

30 En 187/6 av. J.-C., dans le cadre d'une réforme des finances publiques (conséquence indirecte du traité d'Apamée entre Antiochos III et Rome) Philippe V octroya aux régions ainsi qu'aux principales cités de son royaume le droit de frapper monnaie. Sur les types et la chronologie de ce monnayage, qui continue après la fin de la monarchie, voir H. Gaebler, Zur Münzkunde Makedoniens, VI, in ZfN, 36 (1926), p. 111-41, 172; J. Touratsoglou, Macedonia, in A.M. Burnett, M.H. Crawford (éds), The Coinage of the Roman World in the Late Republic, Londres, 1987 (BAR International Series, 326), p. 55-56 et n.14-18 aux p. 62-65 (mise au point détaillée avec bibliographie). Une datation plus haute pour le début de ce monnayage (voir N.G.L. Hammond, in N.G.L. Hammond, F.W. WaLbank, A History of Macedonia III, Oxford, 1988, p. 594) n'est pas étayée par les données numismatiques.

31 La ressemblance de la tête d'Athéna sur certaines émissions macédoniennes du $\mathrm{II}^{\mathrm{e}}$ siècle av. J.-C, avec celle des monnaies athéniennes de «nouveau style » a été relevée par O. Møккноцм, The Chronology of the New Style Coinage of Atbens, in ANSMN, 29 (1984) p. 29-42. Touratsoglou, loc. cil. (n. 30), p. 62-63 n. 16, a montré que c'était aussi le cas des monnaies d'Amphipolis, de Pella et de Thessalonique.

32 Contrairement à ce que pensaient Mørkholm et Touratsoglou (voir la note précédente), un faisceau d'indices numismatiques invite à placer le début du monnayage athénien de « nouveau style » non pas autour de 185 av. J.-C., mais quelque vingt ans plus tard, comme l'ont montré récemment M.R. Price, in A.M. BurnetT, M.H. Crawford (éds), op. cit. (n. 30), p. 95-96, 100 n. 3, et surtout H.B. Mattingly, The Beginning of New Style Attic Coinage, in NumCbron, 150 (1990), p. 67-78, sans doute entre 168/7 et 164/3 av. J.-C. (voir Mattingly, loc, cit., p. 77 n. 43). 
168/7 av. J.-C., lorsqu'Athéna apparaît sur des monnaies de Pella, elle est représentée brandissant la lance, dans un type connu par plusieurs émissions de rois hellénistiques ${ }^{33}$ (voir infra, p. 126-129).

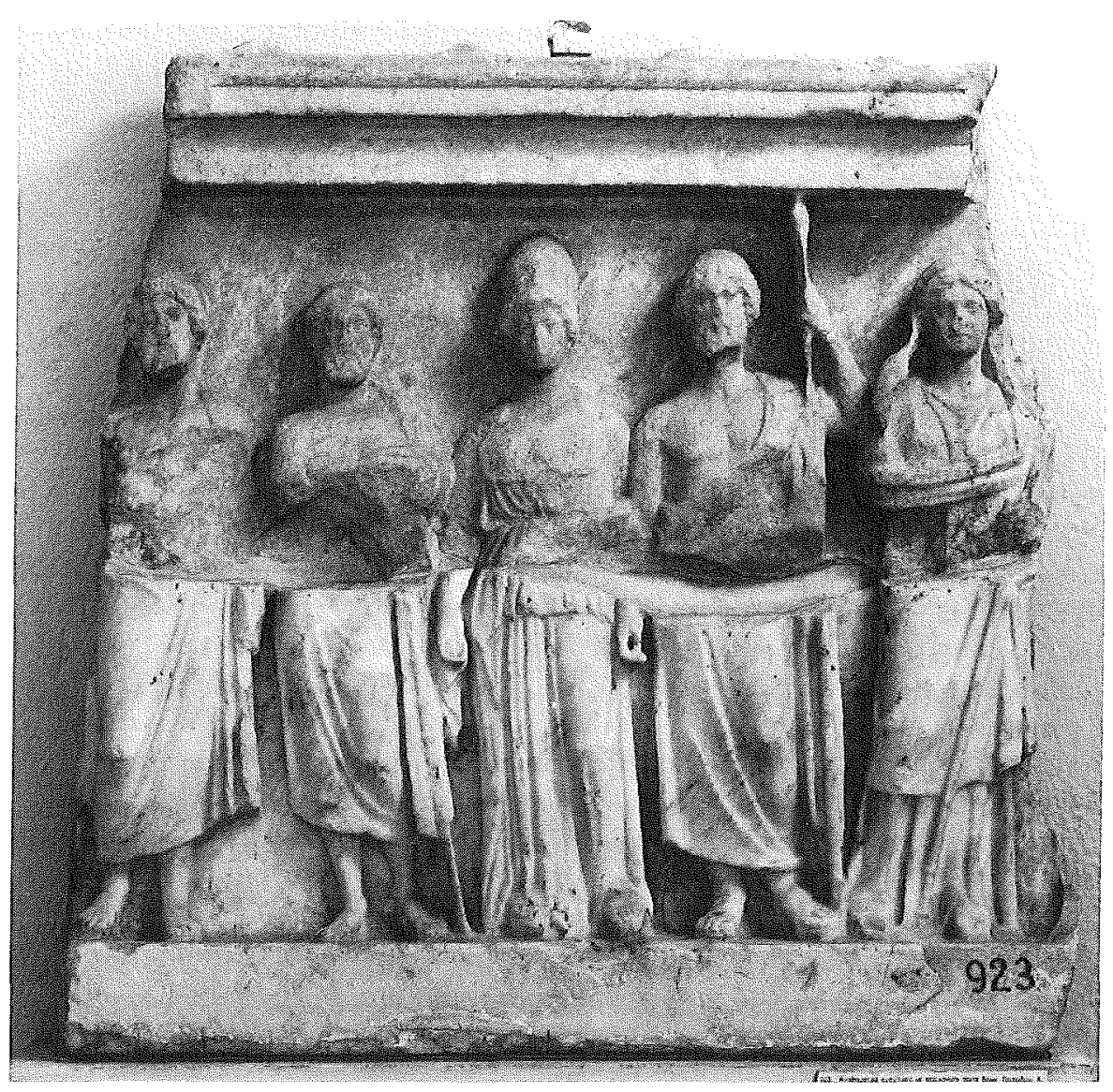

Fig. 6 : Relief votif de Thessalonique. Musée Archéologique de Thessalonique.

Il faut bien noter que le culte d'Athéna est attesté indépendamment du monnayage dans chacune des trois cités. À Amphipolis il existait déjà au v v siècle av. J.-C. ${ }^{34}$, c'est-à-dire bien avant l'annexion de cette ville au royaume de Macédoine, et il remonte sans aucun doute à sa fondation par Hagnon

33 Touratsoglou, loc. cit. (n. 30), p. 55, attire justement l'attention sur les similitudes typologiques et iconographiques entre le monnayage des cités macédoniennes et celui des deux derniers rois Antigonides, ce qui invite à placer la plupart de ces émissions avant 168 av. J.-C.; il observe que la composition des trésors monétaires contenant ces monnaies renforce cette conclusion.

34 Le culte est déjà connu de Thucrdide, V 10, 2. 
comme colonie athénienne. Le sanctuaire n'a pas été découvert jusqu'à présent, mais une inscription, provenant d'Amphipolis et datée de 90/89 av. $\mathrm{J},-\mathrm{C}^{35}$, commémore le couronnement honorifique par un kotvòv $\tau \hat{\omega} v \tau \varepsilon \chi \nu 1 \tau \hat{\omega} v$ d'un collège de quatre prêtres d'Athéna, dont les deux premiers sont à leur tour couronnés par leurs collègues. L'inscription mentionne aussi des noms de vainqueurs aux courses des Jeux isthmiques et olympiques. Il est donc clair que le culte d'Athéna tenait encore une place importante dans la vie publique d'Amphipolis au $\mathrm{I}^{\mathrm{er}}$ siècle av. J.-C.

À Thessalonique, les témoignages épigraphiques et littéraires sur le culte d'Athéna font défaut. Mais un relief votif trouvé à très peu de distance à l'est de l'agora romaine est particulièrement intéressant à cet égard ${ }^{36}$. Dans la partie conservée, on voit cinq divinités représentées de face (fig. 6). Athéna se reconnaît facilement à son casque. À côté d'elle, son père Zeus, le souverain de l'Olympe, est appuyé sur son sceptre. La déesse de l'autre côté de Zeus, qui tient aussi un sceptre et soulève le bout de son himation à hauteur du

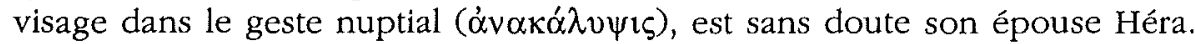
Les deux personages barbus à gauche, bien que difficiles à identifier avec certitude, sont probablement des héros, comme le suggèrent les parallèles iconographiques, avant tout Asclépios et les héros éponymes d'Athènes. Le relief peut être daté, par son style, du dernier quart du Iv ${ }^{e}$ siècle av. J.-C.; il est donc contemporain du synécisme de Thessalonique. Même si nous ignorons quelles divinités étaient représentées dans les parties manquantes, à droite et à gauche, la date du relief, en relation avec sa découverte à proximité du centre civique de la ville, permet de formuler l'hypothèse que la signification de ce document était à la fois religieuse et politique; car les divinités qu'il regroupe semblent bien faire partie du panthéon officiel de la nouvelle cité fondée par Cassandre ${ }^{37}$. Le rôle important d'Athéna dans la vie publique de Thessalonique à l'époque impériale est souligné par la découverte près de l'angle nord-est de l'agora romaine (c'est-à-dire non loin de l'endroit où fut trouvé le relief votif que nous venons d'examiner) des parties en marbre d'une statue colossale acrolithe d'Athéna du type dit de Médicis ${ }^{38}$. Il est inté-

35 L'inscription, conservée aujourd'hui au Musée de Kavalla, a été vue et copiée avant la dernière guerre par $\mathrm{Ch}$. Edson au village de Palaikomi. La transcription d'Edson est reproduite par Ph. Gauthier, M.B. Hatzopoulos, La loi gymnasiarcbique de Béroia,

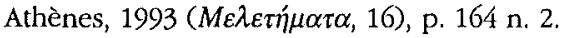

36 G. Despinis, Th. Stefanidou-Tiveriou, Em. Voutiras, Catalogue of Sculptures in the Archeological Museum of Thessaloniki I, Thessalonique, 1997, p. 42-44, $\mathrm{n}^{\circ} 24$ (Em. Voutiras).

37 Sur la datation et l'interprétation de ce document voir Despinis, StefanidouTIVeriou, Voutiras, op. cit. (n. 36), p. 43 (Em. Voutiras).

38 La statue a été étudiée de manière exhaustive et reconstituée en dessin par

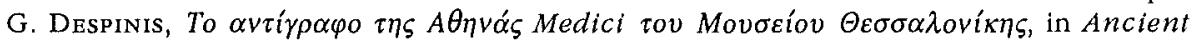
Macedonia II, Thessalonique, 1977, p. 94-102; ID., Aкpó́l $\theta \alpha$, Athènes, 1975, p. 11-16, fig. 1-7, pl. 1-8; Despinis, Stefanidou-Tiveriou, Voutiras, op. cit. (n. 36), p. 97-99, no 72 (G. DesPINIS). 
ressant de noter que cette statue, qui date sans doute de l'époque des Antonins $^{39}$, fut transformée au début du III ${ }^{\mathrm{e}}$ siècle ap. J.-C. en portrait de l'impératrice Julia Domna, épouse de Septime Sévère ${ }^{40}$. Nous ignorons la date exacte ainsi que la raison de cette transformation ${ }^{41}$, mais le fait qu'à Athènes une inscription atteste l'instauration, en 202 ap. J.-C., du culte de Julia Domna comme Athéna Polias ${ }^{42}$, permet de supposer que nous avons affaire à un phénomène analogue. Le culte de Julia Domna a pu être instauré à Thessalonique à l'occasion d'une visite de l'impératrice ${ }^{43}$.

Le cas de Pella est plus difficile. Le seul témoignage littéraire relatif au culte d'Athéna dans cette cité se trouve chez Tite-Live (XLII, 51, 2), qui rapporte, sans doute à la suite de Polybe, comment Persée fut amené à déclarer la guerre aux Romains en 171 av. J,-C. Ayant reçu l'ultimatum du sénat, le roi réunit un conseil à Pella où tous ses généraux se prononcèrent en faveur de la guerre; persuadé que celle-ci était désormais inévitable, il offrit un sacrifice " royal » de cent victimes à Athéna. L'épiclèse de la déesse telle qu'elle est transmise par les manuscrits de Tite-Live est cependant difficile à admettre : ipse centum bostiis sacrificio regaliter Minervae, quam vocant Alcidem, confecto etc. Déjà Turnèbe avait proposé de lire Alcidemon confecto, et cette correction, améliorée en Alcidemon facto par Kreyssig, a été admise par tous les éditeurs récents de Tite-Live ${ }^{44}$. Ainsi a-t-on créé par conjecture une

39 Despinis, loc. cit. (n. 38), in Ancient Macedonia II, p. 99.

40 Les traces de cette transformation sont nettement visibles; elles sont minutieusement décrites par Despinis, ibid., p. 96-99.

41 Despinis, ibid., p. 99-101, propose pour le portrait de Julia Domna une date entre 211 et 217 ap. J.-C.

42 J.H. Oliver, Julia Domna as Athena Polias, in Atbenian Studies Presented to W.S. Ferguson, Cambridge, Massachusetts, 1940 (HSPb, Suppl. vol. I), 521-30 (cf. Bull. épigr. 1946/47, 101); F. Ghedri, Giulia Domna tra oriente e occidente, Rome, 1984, p. 129-131.

43 Une visite de Septime Sévère et de Julia Domna en Macédoine est possible en 202 ap. J.-C.; voir F. Papazoglou, Severa Aurelia Heraclea, in BCH, 85 (1961), p. 162-175. Despinis, loc. cit. (n. 38), in Ancient Macedonia II, p. 100, pense plutôt à une visite de Julia Domna en 214-217 ap. J.-C., lors de la campagne de Caracalla en Orient, en raison de la date tardive qu'il propose pour le portrait.

44 Voir la note de P. Jal dans son édition des livres XLI-XLII de Tite-Live dans la Collection des Universités de France, Paris, 1971, p. 210-11. Les éditeurs plus anciens déduisaient de l'accusatif latinisé Alcidem des manuscrits un nominatif Alkis ( $c f$. Wernicke, in $R E$, I 2 [1894], col. 1550), qui est cependant une forme peu problable pour une épithète d'Athéna, comme l'a remarqué H. GaEbler, Die antiken Münzen von Makedonia und Paionia, II, Berlin, 1936, p. 94, 4. Cf. LACroIx, p. 120 n. 6-7; Kalléris (voir la note suivante), p. 95 n. 2. Parmi les formes attestées la plus proche est le masculin 'A $\lambda \kappa i ́ \delta \eta \zeta$, qui désigne un descendant d'Alkaios. Alcides est une appellation assez commune d'Héraklès (Alkaios était le père d'Amphitryon) dans le langage poétique ( $c f$. Ovide, Métam., IX ,13, 51, 217; XI, 213; XII, 538), ce qui pourrait expliquer la confusion dans les manuscrits de Tite-Live, mais ne saurait justifier l'épithète Alkis, comme ont tenté de le faire Weissenborn-Müller dans leur édition de Tite-Live, Berlin, 1854, ad loc. 
Athéna «Alkidémos », épithète qui signifierait «protectrice des citoyens ${ }^{45}$, et localisé son culte à Pella. Les numismates s'accordent pour reconnaître cette Athéna dans un type monétaire de Pella appartenant à la période 187/6168 av. J.-C. où la déesse est représentée brandissant la lance (fig. 8) ${ }^{46}$. Ce type d'Athéna combattante n'a pourtant aucun caractère local, puisqu'il apparaît constamment sur les monnaies des dynasties hellénistiques (voir infra, p. 126-129).

La découverte à Pella, en 1963, de terres cuites représentant Athéna portant un casque orné de cornes bovines (fig. 7) a confirmé l'existence d'un culte local d'Athéna ${ }^{47}$. La signification des cornes n'est pas claire. Il est tentant de les mettre en relation avec la vache grise $(\pi \varepsilon \lambda \lambda \grave{\eta} \beta$ ôे $)$ à laquelle la ville devait son nom selon une légende locale et qui figure sur les monnaies de Pella à partir du II ${ }^{\mathrm{e}}$ siècle av. J.-C. ${ }^{48}$. Il faut cependant noter que le casque à cornes bovines n'apparaît pas dans les représentations d'Athéna sur les monnaies de la ville. En ce qui concerne le rapprochement de ces statuettes avec la déesse à laquelle Persée sacrifia à la veille de la troisième guerre de Macédoine, il se heurte à une difficulté chronologique, car les terres cuites en question sont de beaucoup postérieures à la fin de la monarchie macédonienne; elles appartiennent toutes à la dernière phase d'occupation de la ville, c'est-à-dire à la fin du II $^{\mathrm{e}}$ et au début du I ${ }^{\mathrm{er}}$ siècle av. J.-C. ${ }^{49}$. Finalement, un examen attentif révèle que la correction de Turnèbe, malgré sa plausibilité paléographique, ne s'impose pas vraiment, car elle ne s'appuie pas sur des parallèles valables : Alkidémos est un nom de personne bien attesté, de formation analogue à Alkimachos par sa première partie et à Aristodémos par

45 F. Solmsen, in Berliner Pbilologiscbe Wocbenscbrift (1907), p. 271; A.B. BRETT, Atbena Alkidemos of Pella, in ANSMN (1950), p. 55-72; J.N. KallérIs, Les Anciens macédo-

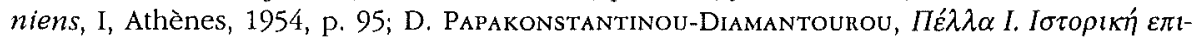

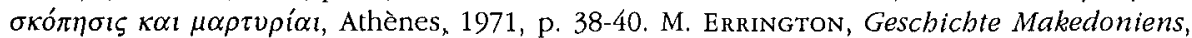
München, 1986, p. 202-203.

46 Pour la date, voir supra n. 33. Le type du droit est une tête de Pan. Le nom "Alkidémos " est souvent donné sans distinction à toutes les Athénas combattantes sur les monnaies hellénistiques; voir infra, p. 127, n. 69. GAEBLER, op. cit. (n. 44), p. 94, pl. XVIII, 27, remarque cependant que seule l'Athéna des monnaies de Pella a droit à ce nom; $c f$. Lacroix, p. 120-21 n. 1. L'exemplaire publié ici appartient à la collection numismatique de Alpha Credit Bank, Athènes.

47 Papakonstantinou-Diamantourou, op. cit. (n. 45), p. 39; I. Touratsoglou, in M.B. Sakellariou (éd.), Macedonia. 4000 Years of Greek History and Civilization, Athènes, 1982, 191, fig. 128; L. KAHIL, in R. Ginouvès (éd.), La Macédoine de Philippe II à la conquête romaine, Paris, 1993 , p. 110, 113 et fig. 97. M. Lilibaki-Akamati, in M. Siganidou, M. Lilibaki-Akamati, Pella (guide arcbéologique), Athènes, 1996, p. 50-51, fig. 32, propose judicieusement de rattacher ces figurines à un culte local d'Athéna protectrice des bovidés, dont l'élevage était pratiqué dans les riches pâturages autour de Pella.

48 Papakonstantinou-Diamantourou, op. cit. (n. 45), p. 39-40, propose prudemment ce rapprochement, en soulignant son caractère hypothétique.

49 Je dois ce renseignement à mon collègue I.M. Akamatis. 


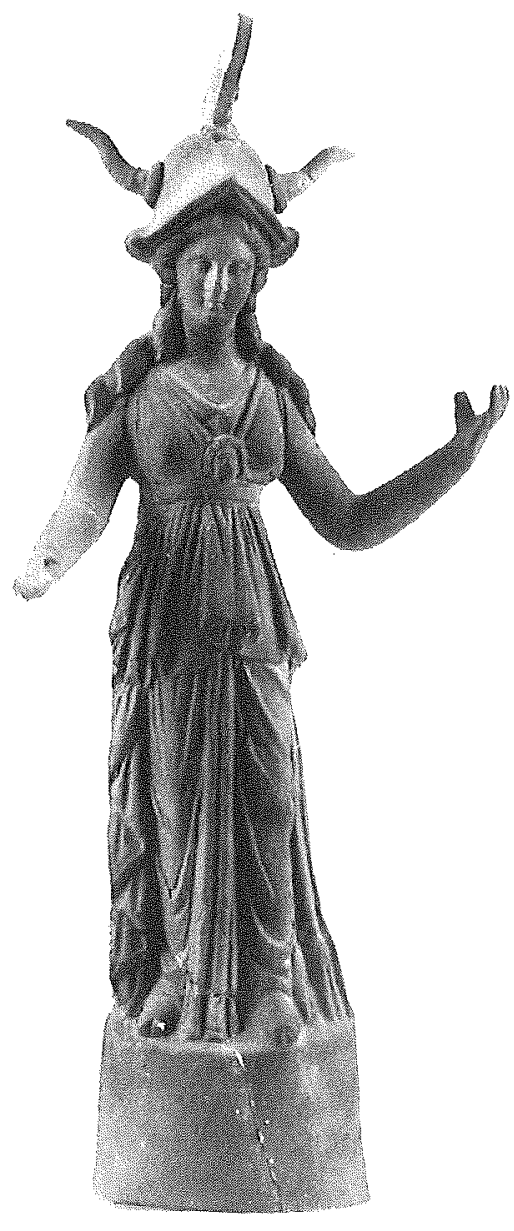

Fig. 7 : Terre cuite de Pella. Musée Archéologique de Pella.

sa seconde partie; la signification du mot $\dot{\alpha} \lambda \kappa \eta$ ( « courage », « valeur ») invite à lui donner le sens de « courageux dans le peuple ». Par contre, comme épiclèse d'une divinité, ce mot composé est difficile à expliquer à cause de sa deuxième partie $(-\delta \eta \mu \circ)$, peu commune dans les épithètes des dieux, surtout en combinaison avec un substantif ${ }^{50}$. Le sens postulé pour l'anthroponyme Alkidémos semble exclure la signification de " protectrice du peuple » qu'on est obligé de lui donner afin de le transformer en épithète acceptable

50 Le cas d'Aphrodite Pandémos est différent, car la première partie de l'épiclèse est un adjectif : c'est l'Aphrodite "de tout le peuple». Sur la signification de l'épithète «Pandémos ", voir WILAMOWITZ, op. cil. (n. 4) I, p. 95. 
d'Athéna ${ }^{51}$. On est tenté de dire que la correction de Turnèbe et de Kreyssig est elegantior quam rectior. L'erreur dans les manuscrits de Tite-Live est sans
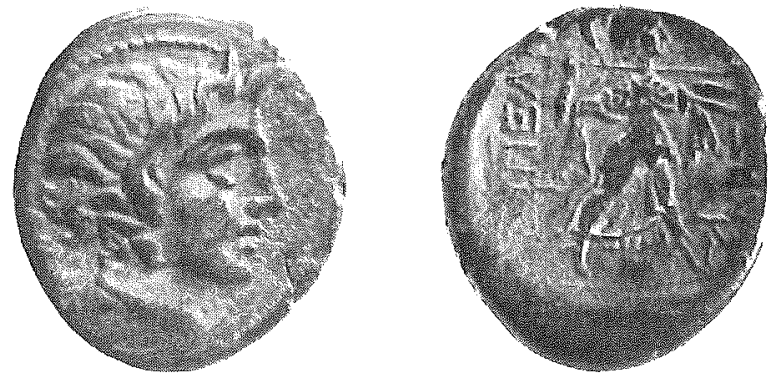

Fig. 8 : Monnaie de bronze de Pella.

doute le résultat de l'ignorance du grec de la part des copistes. En admettant que le texte, tel qu'il nous est transmis, ne s'écarte pas trop de l'original, on a le droit de restituer une épithète déjà attestée d'Athéna, banale (par exemple Alcimachan) ${ }^{52}$, ou rare (par exemple Aloitin) ${ }^{53}$. Quoiqu'il en soit, une Athéna appelée "Alkidémos », inconnue par ailleurs, ne semble pas avoir de place dans le panthéon de Pella - ou dans celui de la Macédoine en général. D'autre part, il est raisonnable de supposer que le sacrifice de cent victimes par lequel Persée scella la déclaration de guerre contre Rome a été offert à une divinité «nationale » plutôt qu'à une déesse locale ${ }^{54}$. C'est sans doute pour cette raison que certains savants ont proposé de rattacher ce sacrifice à un autre sanctuaire d'Athéna, célèbre en Macédoine, celui de Kyrrhos, ville proche de Pella ${ }^{55}$. La renommée de cette Athéna, appelée Kyrrhestis, avait

51 Kalléris, op. cit. (n. 45), p. 95. P. JAL, loc. cit. (n. 44), remarque à juste titre que l'épithète Alkidémos, qui n'est donnée nulle part ailleurs à Athéna, demeure inexpliquée.

52 Cf. Anth. Pal., VI, 124. Cette épithète serait très appropriée pour une déesse de la guerre.

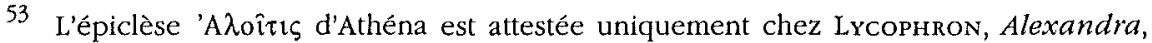
936. La correction Aloitin est très proche de la leçon Alcidem des manuscrits de Tite-Live (une forme latinisée de l'accusatif, Aloitem, pourrait être à l'origine de l'erreur); si on l'adopte, il faudra, bien sûr, garder le participe confecto, comme le faisait Turnèbe.

54 C'est ce que pense Hammond, in Hammond, Walbank, op. cit. (n. 30), p. 511-512, quand il qualifie Athéna Alkidémos de « war goddess of Macedonia ».

55 N.G.L. HAmmond, A History of Macedonia I, Oxford, 1971, p. 159, suivi par P. Goukowsky dans son édition du livre XVII de Diodore de Sicile dans la Collection des Universités de France, Paris, 1978, p. 111 (note à XVII, 4, 5). Hammond ne justifie pas son opinion. De son côté, Papazoglou, op. cit. (n. 7), p. 153 n. 46, remarque à juste titre que le sacrifice mentionné par Tite-Live se rapporte à Pella et non à Kyrrhos. Sur la loca-

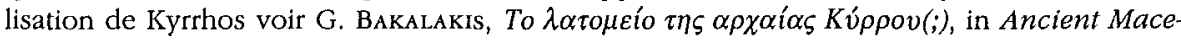
donia I, Thessalonique, 1970, p. 173-183. 
même dépassé les frontières de son pays d'origine, car son culte fut transplanté par des colons macédoniens, avec le nom de Kyrrhos, en Syrie du nord ${ }^{56}$. L'importance du culte de cette déesse est soulignée en outre par le fait que son temple figure parmi ceux qu'Alexandre avait l'intention de construire à grands frais d'après le memorandum ( sa mort ${ }^{57}$. Ce sanctuaire était resté important, du moins au niveau local, jusqu'à l'époque romaine, comme en témoigne une inscription votive à Athéna Kyrrhestis provenant du site même de la ville et commémorant l'affranchissement d'une esclave ${ }^{58}$. Pour en revenir au sacrifice solennel de Persée, le texte de Tite-Live implique qu'il eut lieu à Pella et qu'il fut offert à une Athéna vénérée par les Macédoniens en tant que déesse de la victoire et protectrice de l'armée; son épiclèse, sans doute traditionnelle, a été malheureusement déformée par les copistes. Cette déesse, serait-elle identique à Athéna Kyrrhestis, la plus célèbre de Macédoine? Ce n'est pas exclu, mais rien non plus ne permet de l'affirmer.

Il est clair en tout cas que les rois de Macédoine adoraient Athéna comme divinité guerrière, protectrice de l'armée et d'eux-mêmes. Il ne pouvait en être autrement, puisqu'ils se considéraient comme descendants d'Héraclès, le plus fameux parmi les protégés de la déesse ${ }^{59}$. Athéna donnait la victoire sur le champ de bataille. Aussi est-elle représentée sur le casque et sur la cuirasse du roi Argéade qui était enseveli dans la tombe II du grand tumulus de Vergina, probablement Philippe $\mathrm{II}^{60}$. Athéna était également présente dans le palais royal de Vergina-Aigai, l'ancienne résidence des rois de Macédoine ${ }^{61}$. Lors des fouilles qu'il y effectua en 1861, L. Heuzey découvrit dans la salle circulaire à gauche de l'entrée, appellée «tholos » par les archéologues et qui avait sans doute la fonction d'un sanctuaire domestique ${ }^{62}$, trois menus fragments de reliefs, dont un représentait Athéna ${ }^{63}$. La déesse était en effet

56 Strabon, XVI, C 751, dont dépend StéPh, Byz, s.v. Kúppos; voir Honigmann, s.v.

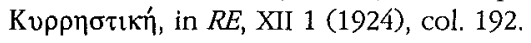

57 Diod. SIC., XVIII, 4, 5.

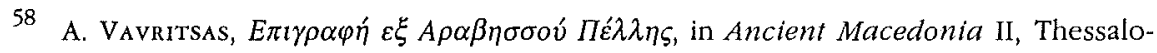
nique, 1977, p. 7-11, fig. 4.

59 Ceci est vrai aussi bien pour les Argéades que pour les Antigonides. Sur les liens de ces derniers avec Héraklès, voir Ch. Edson, in HSPh, 45 (1934), p. 226-232.

60 M. Andronikos, Vergina. The Royal Tombs, Athènes, 1984, p. 137-142.

61 L'identification du site archéologique de Vergina avec Aigai est désormais hors de doute; voir PApazoglou, op. cit. (n. 7) et, tout récemment, M.B. Hatzopoulos, in REG, 109 (1996), p. 264-269.

62 L. Heuzey, Mission archéologique en Macédoine, Paris, 1876, p. 214-219, pl. 14-14b. Le caractère sacré de la "tholos" a été confirmé par la découverte d'une inscription votive à Héraklès Patrôos; voir Andronikos, op. cit. (n. 60), p. 38, 42.

63 Heuzer, op. cit. (n. 62), p. 216, pl. 13. Deux de ces fragments, pl. $13 \mathrm{n}^{\mathrm{os}} 1-2$, sont aujourd'hui au Louvre; celui avec la figure d'Athéna est connu seulement par le dessin de H. Daumet publié dans l'ouvrage de Heuzey, pl. $13, \mathrm{n}^{\circ} 3$. 
considérée comme protectrice des rois macédoniens. La campagne d'Alexandre en Asie fut placée dès le début sous la protection d'Athéna. Au point où il traversa l'Hellespont, le roi érigea trois autels : un à Zeus $\alpha \pi$ o $\beta \alpha \tau \eta \dot{\eta} \rho 1 o \zeta$, un à Athéna et un à Héraklès ${ }^{64}$. Alexandre visita ensuite le sanctuaire d'Athéna Ilias sur l'acropole de Troie, y dédia son armure et emporta des boucliers anciens, offerts selon la tradition par les Grecs victorieux des Troyens, qu'il faisait porter devant son armée dans les batailles ${ }^{65}$. Le temple d'Athéna Ilias était parmi ceux dont il avait prévu la reconstruction (voir supra n. 57). Après sa première victoire au fleuve Granikos il dédia au plus célèbre de tous les sanctuaires de la déesse, celui de l'Acropole d'Athènes, les armes prises à l'ennemi ${ }^{66}$.
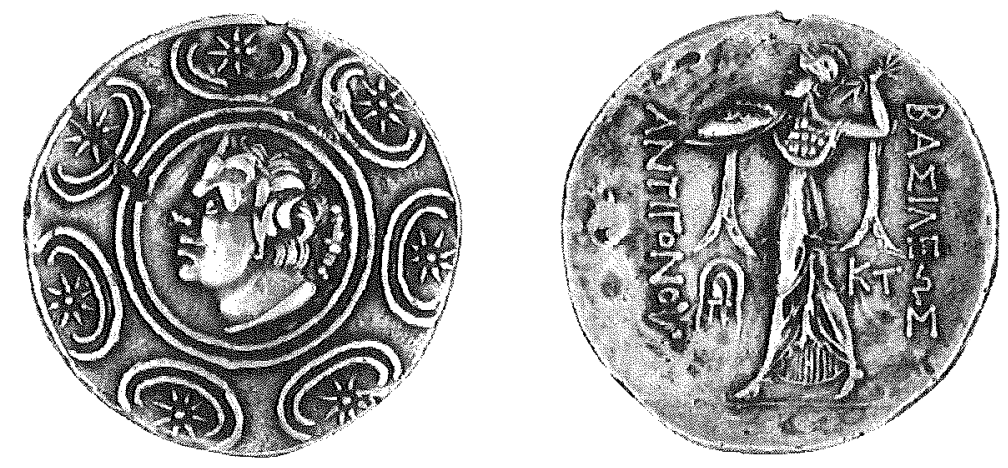

Fig. 9: Monnaie d'argent d'Antigone Gonatas.

Pour les diadoques aussi Athéna était la déesse de la victoire. Ceci apparaît clairement dans leur monnayage. On sait qu'après la mort d'Alexandre, ses successeurs continuèrent à frapper des monnaies portant son nom et son portrait avec, au revers, la figure de Zeus assis ${ }^{67}$. Le premier diadoque qui introduisit un type différent au revers des monnaies au nom d'Alexandre fut Ptolémée, alors encore officiellement gouverneur d'Égypte : sur les nouvelles monnaies d'argent introduites en $315 / 4$ av. J.-C., on voit l'image d'Athéna brandissant la lance ${ }^{68}$. Le type choisi, de style nettement archaïsant, semble conçu pour le dessin et le relief plutôt que pour la sculpture en ronde bosse;

64 ARriEN, Anab., 1, 11, 7.

65 Arrien, Anab., I, 11, 7-8; cf. A.B. BosworTh, A Historical Commentary on Amian's History of Alexander. Volume I. Commentary on Books I-III, Oxford, 1980, p. 102.

66 Arrien, Anab., I, 16, 7; cf. Bosworth, op. cit. (n. 65), p. 127.

67 O. MøвкHOLM, Early Hellenistic Coinage from the Accession of Alexander to the Peace of Apamea (336-186 B.C.), Cambridge, 1991, p. 55-62.

68 O. Mф̆ккнодм, op. cit. (n. 67), p. 26, 64-65, fig. 92. Sur la date d'introduction du nouveau type monétaire par Prolémée, voir B. Kuschel, Die neuen Münzbilder des Ptolemaios Soter, in $J N G, 11$ (1961), p. 9-18, en particulier p. 17-18. 
il est sans doute inspiré des représentations d'Athéna Promachos sur les amphores panathénaïques ${ }^{69}$. On y retrouve la même attitude et le même maniérisme dans le rendu des vêtements, surtout de l'bimation court porté sur les épaules, dont les pans prennent la forme de queues d'hirondelle. Cette Athéna fait déjà son apparition, comme type secondaire, sur des monnaies d'Alexandre frappées à Amphipolis en $326 / 5$ av. J.-C. ${ }^{70}$. La valeur symbolique de l'image d'Athéna combattante est mise en évidence par le fait qu'elle est reprise sur les monnaies de Démétrios Poliorcète après sa victoire navale contre Ptolémée à Salamine de Chypre, en 306 av. J.-C. ${ }^{71}$. De nombreux rois hellénistiques adoptèrent dans leur monnayage le même type d'Athéna ${ }^{72}$ : Séleucos I, Pyrrhos, Antigone Gonatas (fig. 9) ${ }^{73}$, Philippe V (fig. 10) ${ }^{74}$. Sur les monnaies des deux derniers, Athéna se distingue en plus par un attribut qui la rapproche davantage de son père Zeus : le foudre. L'image de la déesse brandissant le foudre avait peut-être semblé préférable aux rois Antigonides parce qu'elle se distinguait de la Promachos traditionnelle à la lance ${ }^{75}$. Cette Athéna se retrouve jusqu'aux confins du monde hellénique, en Bactriane et en Inde, puisqu'elle figure sur les émissions du roi indo-grec Ménandre, vers

69 Le rapprochement de cette Athéna avec celle des amphores panathénaïques a déjà été fait par LACrorx, p. 116-121, dont la discussion reste fondamentale. Sur l'Athéna archaissante des monnaies hellénistiques voir C.M. HAvelock. The Archaïstic Athena Promachos in Early Hellenistic Coinages, in AJA, 84 (1980), p. 41-50; M.-A. ZAGDoun, La sculpture archaisante dans l'art bellénistique et dans l'art romain du Haut-Empire, Paris, 1989 (BEFAR, 269), p. 53-57; MфккноLм, op. cit. (n. 67), p. 26. Dans le passé, certains savants ont voulu reconnaître dans cette figure une représentation de la prétendue Athéna "Alkidémos » de Pella, dont nous avons parlé supra, p. 121-123; cf. BretT, op. cit. (n. 45). Malgré la mise en garde de GaEBler (voir supra n. 46), cette identification infondée est reprise dans de nombreuses études, même récentes. L. LACroix, in BCH, 73 (1949), p. 168, a d'ailleurs observé que ce type archaïsant d'Athéna est si répandu qu'il est impossible d'y reconnaître une statue locale; $c f$. ZAGDOUN, op. cit., p. 57.

70 Lacrolx, p. 116 n. 2; Zagdoun, op. cit. (n. 69), p. 54-55, pl. 3 fig. 13.

71 MøкKноLм, op. cit. (n. 67), p. 26, 77-78. Le symbolisme de la victoire est évident sur le type du droit qui représente une Niké debout sur une proue de navire sonnant le clairon. La même victoire navale de Démétrios fut également célébrée par une émission à la figure de Poseidon.

72 Voir les remarques de Mørкноцм, op. cit. (n. 67), p. 26.

73 Mørкholm, op. cit. (n. 67), p. 134. La tête de Pan sur le droit de ces monnaies est une référence à la bataille de Lycimacheia, où ce dieu sema la panique parmi les gaulois. L'exemplaire publié ici appartient au Musée Numismatique d'Athènes: I. Touratsoglou, Disjecta membra, Athènes, 1995, p. 93, nº 19.

74 MøвкноLм, op. cit. (n. 67), p. 136. Ces monnaies datent probablement du début du règne de Philippe V. L'exemplaire publié ici appartient à la collection numismatique de Alpha Credit Bank, Athènes.

75 Sur les différentes tentatives d'interprétation d'Athéna au foudre, voir A. B. CoOK, Zeus, III 1, Cambridge, 1940, p. 868-873. Selon J. Nollé, in JNG, 45 (1995), p. 56-63, l'image d'Athéna brandissant le foudre serait inspirée de la gigantomachie et symboliserait la victoire des Grecs contre les barbares. 
le milieu du $\mathrm{II}^{\mathrm{e}}$ siècle av. J.-C. (fig. 11) ${ }^{76}$ ainsi que sur celles des derniers rois indo-grecs (Zoïlos II, Dionysios, Apollophanès, Straton II) ${ }^{77}$.
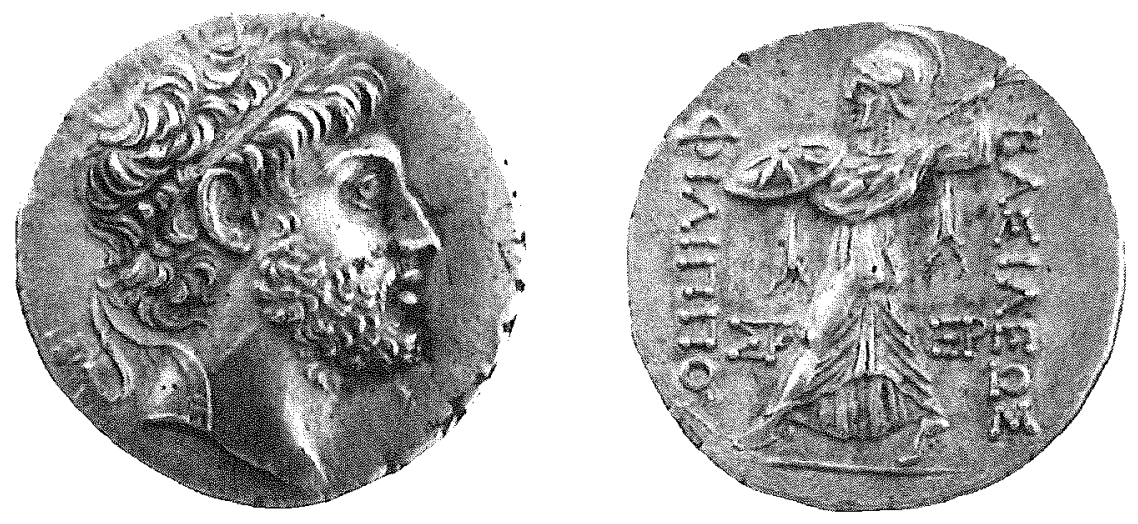

Fig. 10 : Monnaie d'argent de Philippe V.
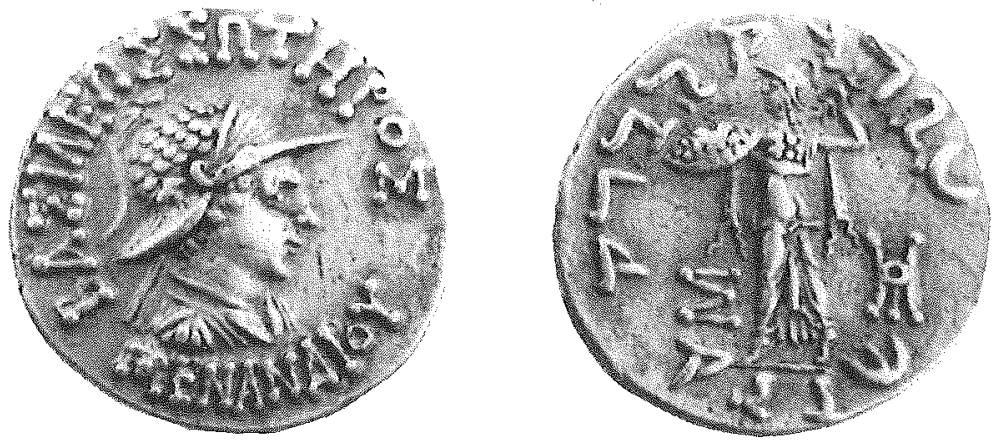

Fig. 11 : Monnaie d'argent de Ménandre.

Dans l'ensemble, Athéna semble avoir été, en Macédoine comme ailleurs, principalement une divinité du domaine public. Son culte avait dans la plupart des cas une signification politique. La déesse elle-même pouvait avoir la fonction d'un symbole. Mais le sens qu'on lui donnait n'était pas toujours le même. À l'intérieur des cités, Athéna apparaissait comme divinité poliade, garante des libertés civiques. Seul le culte d'Athéna Kyrrhestis semble avoir

76 O. BopearachCHI, Monnaies gréco-bactriennes et indo-grecques. Catalogue raisonné, Paris, 1991, p. 86-87. L'inscription du revers est en alphabet kharoshthi; $c f$. Bopearachchi, ibid., p. 452. L'exemplaire publié ici appartient à la collection numismatique de Alpha Credit Bank, Athènes.

77 Bopearachchi, ibid., p. 85. 
dépassé sa ville d'origine. Pour les rois, la déesse était avant tout protectrice de l'armée et porteuse de victoire. Le fait qu'en Macédoine sous la monarchie la vie politique des cités était étroitement contrôlée par les souverains enlevait peut-être aux cultes locaux d'Athéna une partie de leur importance. Mais, chaque fois que les circonstances le permettaient, les cités mettaient son culte en valeur pour affirmer leur autonomie.

Université de Thessalonique

Emmanuel Voutiras

Département d'archéologie et d'histoire de l'art

GR - 54006 THESSALONIQUE 